\title{
The spatial and temporal assessment of clustered and time-dependent seismic responses to mining
}

\author{
K Woodward Australian Centre for Geomechanics and The University of Western Australia, Australia \\ J Wesseloo Australian Centre for Geomechanics and The University of Western Australia, Australia \\ Y Potvin Australian Centre for Geomechanics and The University of Western Australia, Australia
}

\begin{abstract}
The phenomenon of seismicity is observed in many hard rock underground mines around the world. The potential for seismic events to damage underground excavations can create a significant hazard to mining personnel, equipment, and infrastructure. The management of seismic hazard is an essential component in minimising the political, social, and economic risks associated with mining.

The effective management of seismic hazard is underpinned by a sufficient understanding of the magnitude, spatial, and temporal characteristics of seismicity. These characteristics of seismicity are controlled by causative seismic source mechanisms within a mine and are related to stress conditions, rock mass strength, excavations, geology, and geological features. This paper considers spatially clustered seismicity, which is generated by a time-dependent rock mass failure process. Seismicity of this nature is routinely observed following blasting or large seismic events and is referred to as a 'seismic response' within this paper.

There are numerous interrelated factors that can influence the characteristics of seismic responses and this makes it difficult to establish meaningful correlations with causative processes. Furthermore, the management of seismic response hazard has the tendency to rely on the site-specific experience, which has inherent limitations. These areas of research can be partly addressed by the quantification of seismic responses that allows for the development of an objective understanding of seismic response hazard.

This paper presents a general outline of a recently published methodology for the assessment of seismic responses that concurrently examines the spatial and temporal characteristics of these responses. A general discussion on the major considerations when applying the method is provided in this paper. The benefits of the quantification of seismic responses are illustrated by several case studies. These studies assess individual responses and consider the historical distribution of response characteristics for a mining environment.
\end{abstract}

Keywords: seismicity, short-term, response, spatial, temporal, delineation, quantification

\section{Introduction}

There are two broad categories of seismic events associated with mining that are synonymous with the characteristics of earthquakes (Kagan \& Jackson 1991; Utsu 2002; Hudyma 2008; Plenkers et al. 2010). Firstly, seismic events that typically follow a stationary Poisson process and are referred to as background or time-independent seismicity. Secondly, a heightened rate of spatially-related events that may occur over relatively short periods and typically follow a non-stationary Poisson process, e.g. seismicity that occurs after blasting or large seismic events. This paper focuses on the assessment of this second category and refers to spatially and temporally-related events as a seismic response.

Mining seismicity is influenced by many factors that contribute to the rock mass excitation and relaxation processes and it is the interplay between these factors that contributes to the complexity of results and the difficulty in establishing long-term correlations between seismicity and underlying physical processes (Mendecki \& Lynch 2004; Mendecki 2005). 
Literature indicates that the variability associated with seismic responses can be attributed to several factors. These factors are (Malek \& Leslie 2006; Heal 2007; Eremenko et al. 2009; Kgarume et al. 2010b; Penney 2011; Vallejos \& McKinnon 2011):

- The variability of natural and induced influences on seismicity, for example the principal stress conditions, mining geometries, rock mass characteristics, and blasting.

- The inability to quantify influencing factors, for example the use of depth or volume mined as a proxy for stress conditions.

- An insufficient understanding of the physical processes that result in seismic responses, for example, causative stress transfer processes.

The study of seismic response characteristics has significant implications for mining operations where seismicity is a source of hazard for the workforce and infrastructure. The potential for seismic risk to adversely affect mining operations and the requirement for the mining industry to continually improve the management of seismic hazard is illustrated by the 2006 Anzac Day event at the Beaconsfield gold mine (Hills \& Penney 2008). In addition to significant economic loss, this event resulted in the loss of one life and a 14-day operation to rescue two trapped miners. The extensive review following this tragedy highlighted the importance of improving methods of managing seismic response hazard through the use of re-entry protocols (Melick 2007).

Hudyma (2008) and Larsson (2004) note that local experience from ground control engineers and management forms the basis of workplace closure and re-entry practices. This is supported by Vallejos and McKinnon (2008) who found, in a survey of 18 seismically active mines, that re-entry protocols in over $70 \%$ of these mines were based on local experience. Although valuable insight gained through site experience is important in managing seismic hazard, there are clear dangers associated with relying too heavily on unquantified personal impressions and interpretations. Mendecki (2008) discussed this topic in a broader context of seismic hazard, suggesting that the use of human judgment to reduce complexities in the assessment may be subject to limitations of experience, interpretation, and motivational biases.

Identification and delineation of seismic responses allows for the quantitative assessment of time-dependent seismicity. This work aims to offer a method to consistently quantify seismic responses in order to gain insight into causative rock mass failure processes, optimise the reliability and operational aspects of re-entry protocols, and reduce subjectivity of engineering decisions.

\section{Delineation of seismic responses in space and time}

This paper provides an overview of the method used to identify and delineate seismic responses in space and time. Further details of the method can be found in Woodward (2015). This work also incorporates an assessment of the method's performance including metrics to quantify spatial clustering and common errors associated with temporal modelling of seismic responses, e.g. responses with early temporal variation, short seismic responses, and the inclusion of time-independent seismicity. The process for delineating seismic responses used in this paper is iterative with four distinct steps. The general overview of this procedure is illustrated in Figure 1 and an example of the method's practical application is provided in Figure 2.

Iterative Approach to Delineation of Seismic Responses

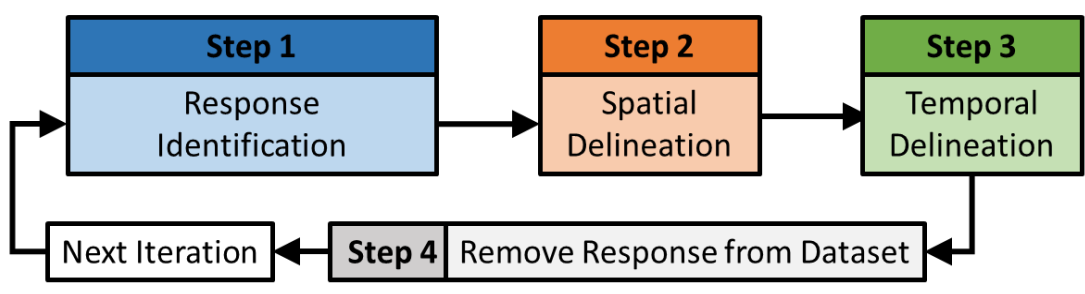

Figure 1 The framework for response identification, spatial delineation, temporal delineation and response removal 
Step 1: Identification

Step 2: Spatial Delineation
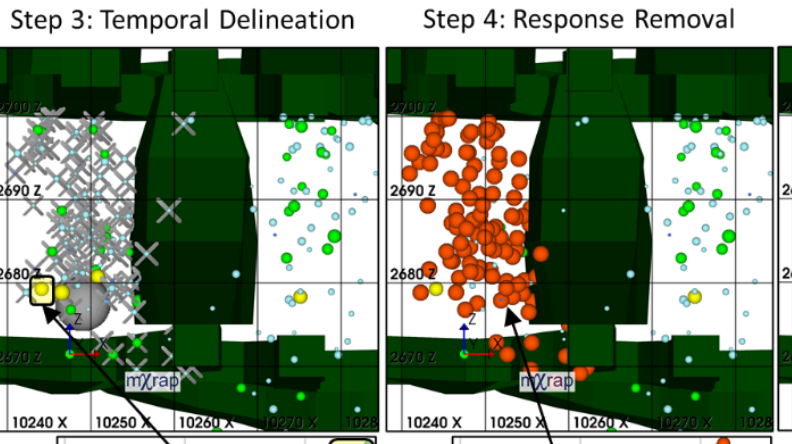

Additional Iteration
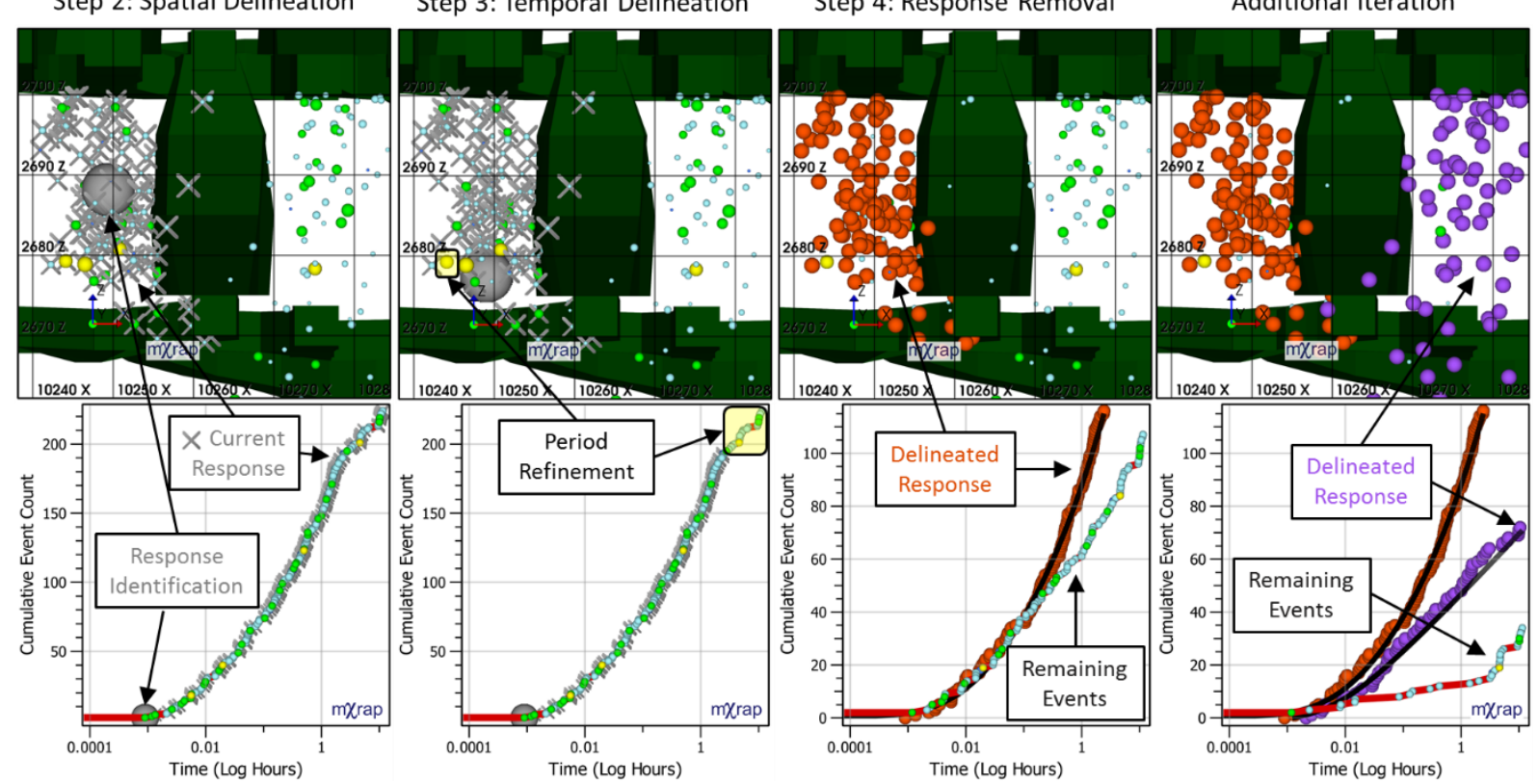

Figure 2 Practical application of the method to identify and delineate a seismic response

Step 1. A seismic response is identified in space and time by a heighted rate of seismic event occurrence, which are also spatially clustered. Initial response identification is defined as the first event in the response and the mean location of all response events. This time and location is represented by the grey sphere in the first frame of Figure 2 .

Step 2. The information provided by response identification is used to spatially delineate associated events using a density-based clustering algorithm. These events are represented by grey crosses in the first frame of Figure 2.

Step 3. Temporal modelling delineates a period of consistent time-dependent behaviour for the spatially-delineated events (grey sphere). These events are represented by grey crosses in the second frame of Figure 2. The modelling period (and response events) is refined due to inconsistencies late in the response (highlighted in the time series).

Step 4. The delineated response is removed from the dataset (orange spheres in the third frame of Figure 2). The procedure is iteratively re-applied to the remaining events. An additional response is identified, delineated and removed in the fourth frame in Figure 2 (purple spheres).

\subsection{Response identification}

Seismic responses are indicated by heightened rates of seismic events in space and time. A measure of the spatial and temporal rates of seismicity can be achieved by assessing each seismic event with respect to its number of subsequent neighbouring events. Response identification considers event occurrence with respect to a spatial window ( $\mathrm{S}_{\mathrm{w}}$ : the maximum Euclidean distance to neighbouring events), temporal window ( $T_{W}$ : the time period to assess subsequent events), and count threshold $\left(C_{T}\right.$ : the number of neighbouring and subsequent events required to identify the beginning of a seismic response). The subsequent neighbours are defined using spatial and temporal windows that are characteristic of individual processes and their scales that exist within the mining environment, e.g. development blasting, production blasting, and delocalised processes. The site-specific configuration of the seismic monitoring network must be considered to limit the analysis to only assess seismic events that are consistently and accurately observed, i.e. event location precision and accuracy, along with the minimum event magnitude that can be consistently recorded. 
The measurement of spatial and temporal event rates can be implemented within an algorithm that iteratively identifies seismic responses. The initiation time of a response is identified as the timing of the event with the most subsequent neighbours (in space and time) and the location of the response is taken as the mean location of those events. The response time and location are later adjusted to reflect the delineated response. The seismic response is then spatially and temporally defined and removed from the dataset. After removal of the response, the entire procedure is then repeated on the remaining data, i.e. the event with the most subsequent neighbours in the remaining data indicates the occurrence of the next response to be considered.

\subsection{Spatial delineation}

The spatial characteristics of mining seismicity are controlled by the factors that influence the rock mass failure process. Assessing the spatial event distributions is the basis for understanding and quantifying seismic hazard (Wesseloo 2014). A clustering procedure can be applied to spatially isolate events that are associated with individual seismic source mechanism, although most previous studies consider additional event characteristics within clustering procedures. Examples of this include Hudyma (2008), Malek and Leslie (2006), Frohlich and Davis (1990), Falmagne (2001), Cho et al. (2010), and Rebuli and Kohler (2014).

The most fundamental aspect of clustering procedures is that elements (i.e. seismic events) which share similar characteristics are grouped together and the underlying structures present within a dataset are identified (Jain et al. 1999). It is important to identify which seismic event characteristics are shared and, hence, form the basis for clustering. For this application, clustering aims to delineate spatial clusters of events and only considers event location to minimise assumptions concerning the shared characteristics of a seismic response.

A simple density-based method is Density-Based Spatial Clustering of Applications with Noise (DBSCAN), proposed by Ester et al. (1996), which provides the general framework for such approaches. The DBSCAN approach classifies elements as a core, boundary, or noise element by considering the number of neighbouring elements $\left(N_{e}\right)$ with respect to a user-specified minimum $\left(N_{\text {MIN }}\right)$ within a search distance $\left(D_{s}\right)$. DBSCAN creates clusters from adjacent core elements and their neighbours. Core events are recursively considered and merged if one or more core elements are within $D_{S}$ (Ester et al. 1996).

Density-based clustering is a non-parametric method suited to mining seismicity as it allows for distinct class identification, has minimal requirements of existing dataset knowledge, enables the discovery of clusters with arbitrary shapes, and permits the discovery of high-density clusters within low-density areas (Ester et al. 1996; Kriegel et al. 2011).

When applied to mining seismicity, density-based methods have several shortcomings adversely affecting their performance. These include the necessity of finding a suitable density threshold, a poor performance for datasets with varying element densities, and the sensitivity of clustering outcomes to clustering parameters.

We introduced two modifications to the DBSCAN procedure to ensure that the method is generally applicable to mining seismicity. These modifications focus on addressing limitations of clustering datasets of varying densities and the sensitivity of outcomes to parameter selection.

The first modification integrates the density-based method into an iterative framework that provides a structured approach to assessing clusters. The framework considers the event with the most neighbours, i.e. the volume of highest event density for the given time interval. The method then clusters events that have a similar number of neighbouring events. Similar densities are defined to be within user-specified percentage of highest event density. The delineated cluster is removed from the dataset before considering the next event with the most neighbours. This process is repeated until no events remain in the dataset that have more than an absolute minimum number of neighbours within the specified search distance. Furthermore, this entire process is then repeated using increasingly larger search distances, which allow the identification of relatively sparser clusters once the denser clusters have been removed. This modification addresses DBSCAN's shortcomings when attempting to cluster datasets of varying densities.

The second modification is to utilise the neighbouring event density initially found by the first modification. This defines the density required for events to be considered as part of the core. The initial estimate will 
always correspond to the highest density in the dataset and, hence, a tolerance must be applied to the initial upper estimate. The tolerance allows core events to be more leniently defined and the cluster to spread from the densest region. This estimate is refined as additional core events are discovered for each individual cluster. This modification replaces the user-specified minimum number of neighbours $\left(\mathrm{N}_{\mathrm{MIN}}\right)$ with a spatial density tolerance $\left(\mathrm{S}_{T}\right)$ and addresses DBSCAN's sensitivity of clustering outcomes to parameter selection.

\subsection{Temporal delineation}

The most widely used model for describing time-dependent occurrence of earthquake aftershocks is the modified Omori Law (MOL), (Omori 1894a, b; Utsu 1961). Utsu (1961) designated the altered equation as the modified Omori formula, which is written as Equation (1).

$$
n(t)=K(t+c)^{-p}
$$

where:

$$
\begin{array}{ll}
\mathrm{n}(\mathrm{t}) & =\text { number of events per time interval. } \\
\mathrm{t} & =\text { time. } \\
\mathrm{p} & =\text { constant (rate of decay). } \\
\mathrm{K} & =\text { constant (productivity) } \\
\mathrm{c} & =\text { constant (time offset) }
\end{array}
$$

The MOL was proven to be applicable to earthquake aftershocks (Utsu et al. 1995) and this model is generally applied to mining seismic responses (Kgarume 2010; Vallejos \& McKinnon 2010a), although, not all responses are well described by the MOL (Mendecki \& Lynch 2004). There is no clear preferable model despite alternatives being applied to earthquake aftershocks and responses to mining, e.g. exponential distribution, stretched exponential distribution, and epidemic type aftershock model (Kisslinger 1993; Gross \& Kisslinger 1994; Gasperini \& Lolli 2009; Vallejos \& McKinnon 2010a).

There are fundamental aspects of mining seismicity that diverge from natural earthquake seismicity that invalidate some models. For example, epidemic-type models do not take into account contributions to seismic productivity from blasting. Seismic responses are often subjected to additional time-dependent influences from routine mining activities, e.g. blasting, or mucking/bogging, and are not expected to be precisely described by a simple model. The most common example is a seismic response being influenced by a subsequent blast or large seismic event. The new seismic response appears to truncate or superimpose with the previous response, and may include some portion of time-independent seismicity. There is inherent uncertainty concerning to what degree the recent blasting or seismic events have altered time-dependent failure processes. Currently, there are no obvious benefits to considering more complex temporal models to quantify seismic responses due to this uncertainty. These concerns are partially addressed by considering the spatial characteristics of the seismic response, which are vital to isolate seismicity generated by distinct rock mass failure processes. Furthermore, information is gained if a seismic response cannot be suitability modelled. Divergence in temporal event characteristics may indicate that additional unexpected processes are contributing to seismicity, although confidence in this interpretation is site-specific and related to the consistency in spatial and temporal modelling of historical responses.

\subsubsection{MOL parameterisation}

Ogata (1983) provided a maximum likelihood method to estimate the MOL parameters (Equation (1)) and their associated uncertainties ( $\mathrm{p \sigma}, \mathrm{K} \sigma$, and $\mathrm{c} \sigma$ ). Maximum likelihood estimates (MLE) are obtained by maximising a log-likelihood function over a modelling interval.

The p-parameter is proportional to the decay rate of seismic responses. Values for mining seismic responses range from 0.4 to 1.6 (Spottiswoode 2000; Kgarume et al. 2010a; Vallejos \& McKinnon 2010a) and agree with 
typical p-values from the study of earthquakes (0.5-1.5) (Dieterich 1994). There are no definitive factors influencing the decay rate of mining seismic responses (Vallejos \& McKinnon 2010b).

The K-parameter is proportional to the productivity of a seismic response and is expressed on an arbitrary scale due to the variable sensitivity of seismic monitoring. Response productivity was correlated to the mainshock magnitude for two South African mines (Kgarume et al. 2010b), along with the volume blasted and mining depth for the Canadian case studies (Vallejos \& McKinnon 2011). In contrast, Kwiatek (2004) observed a significant variation in mining seismic responses and found no correlation between mainshock magnitude and response productivity.

The c-parameter has no physical meaning but has two important functions. Firstly, to avoid the mathematical divergence when time is zero for the MOL (Molchan \& Dmitrieva 1992; Gross \& Kisslinger 1994). Secondly, the c-parameter accounts for early aftershock deficiency (EAD). This is a phenomenon where temporal observations are inconsistent as too few events are observed early in the response. EAD is most commonly attributed to monitoring and waveform processing constraints (Spottiswoode 2000; Kagan 2004, 2006; Helmstetter et al. 2005; Enescu et al. 2009; Kgarume 2010). There is a positive correlation between the c-parameter and p-parameter due to an interdependency of these parameters within the MLE procedure (Gasperini \& Lolli 2006). The influence of interdependency between these two parameters is addressed by the selection of an appropriate temporal modelling interval, which is discussed in Section 2.3.2 and covered in more detail in Woodward (2015).

\subsubsection{Application of the MOL to mining seismic responses}

The application of temporal modelling requires fundamental decisions in selecting a subset of sequential seismic events and the definition of relative event times. The application of the $\mathrm{MOL}$ to mining seismic responses and the resultant temporal delineation of events is approached as an optimisation problem, which considers a statistical comparison of different modelling intervals. Approaches to selecting a modelling interval vary between studies and can have a significant impact on the applicability of the MOL, the determination of the MOL parameters, and their uncertainties (Narteau et al. 2002). The definition of the principal seismic event and the c-parameter must be allowed to vary in order to achieve a consistent statistical comparison of different modelling intervals (Woodward 2015). The c-parameter is ideally zero to mitigate potential bias to the $\mathrm{p}$-parameter, although this parameter may be required to account for periods of EAD due to inherent monitoring limitations.

The MLE typically forms the basis for modelling selection through its incorporation into an information criteria (Akaike 1974). Nyffenegger and Frohlich (1998) noted that low uncertainties in the MOL parameters did not indicate model unsuitability. Therefore, the selection of an appropriate modelling interval cannot be achieved by the optimisation of only an information criteria based on the MLE. The non-parametric Anderson-Darling (AD) statistic assesses the absolute suitability of model fit (Anderson \& Darling 1954; Lewis 1961). This statistic is accompanied by guidelines relating to fit quality and numerical values, e.g. 0: perfect fit, 1: good fit, 2: acceptable fit, and > 2: unacceptable fit (Lewis 1961; Nyffenegger \& Frohlich 1998, 2000; Vallejos \& McKinnon 2010a). The AD statistic is sensitive to misfit data early and late in the response, and is an informative criterion when selecting appropriate modelling intervals (Nyffenegger 1998).

The suitability of a modelled interval is partially indicated by low uncertainty in MOL parameters. This can be represented by the amount of standard deviation in the marginal error, relative to absolute $\mathrm{p}$ and K-values i.e. $\left(\frac{p \sigma}{p}+\frac{K \sigma}{K}\right) / 2$. Lower parametric uncertainty is achieved as the average of these values approaches zero.

The c-parameter, average portion of standard error, and AD statistic provide three measures that form the basis for the selection of an optimal modelling interval. These measures do not capture additional information concerning the acceptable range of parameter values that are required to place emphasis on ideal solutions, limit the influence of poor solutions, or provide proportional scaling within a range of marginal parameter values. The absolute additional information associated with each relative parametric and statistical measure can be incorporated by undergoing a continuous piecewise linear transformation. 
The weighted factors that measure the quality of the MOL modelling must be combined in a holistic decision metric that can be optimised. The delineation objectives are incorporated by considering the MLE and the number of events during each possible modelling interval. This prevents a large number of events from being excluded from the temporal model for a marginal benefit to the weighting of modelling parameters. The ideal solution will exhibit high parameter weightings ( $S E_{w}, C_{w}$, and $A D_{w}$ ), a large number of events ( $N_{\text {Events }}$ ), and a high MLE. A multiplicative function heavily penalises a solution if any individual measure is relatively small. Due to this function's simplicity, it also offers pragmatic benefits in construction, application, and interpretation (Equation (2)).

$$
M L E_{W}=s e_{W} \times A D_{W} \times c_{W} \times N_{\text {Events }} \times M L E
$$

where:

$$
\begin{aligned}
& M L E_{W}=\text { weighted maximum likelihood estimate metric. } \\
& \text { sew }_{W}=\text { standard error weight. } \\
& A D_{W}=\text { Anderson-Darling weight. } \\
& C_{W}=\text { c-parameter weight. } \\
& N_{E v e n t s}=\text { number of events within the modelling interval. } \\
& M L E=\text { maximum likelihood estimate. }
\end{aligned}
$$

\section{Case studies}

The case studies presented within this paper aim to illustrate the implementation, typical results, and benefits of quantifying seismic responses. These examples have two focuses. Firstly, case studies of individual or multiple seismic responses occurring over a period of days, and secondly, results from the assessment of seismic data for an entire mining environment. The seismic data used within these examples has undergone routine quality and consistency filtering to exclude events smaller than magnitudes that can be consistently recorded or have clearly erroneous source parameters or location.

\subsection{Assessment of an individual seismic response}

The following case example presents analysis results from the delineation of an individual seismic response within an open stope mine over a short time period. Spatial clustering uses a $D_{S}$ of $10 \mathrm{~m}$ and $\mathrm{S}_{\mathrm{T}}$ of $50 \%$. This seismic response of 192 events was well modelled by the $\mathrm{MOL}(A D=0.55)$ with a nominal decay rate $(p=1.04)$, productivity $(K=22.24)$, and a minimal c-parameter $(c=0.008)$. Figure 3 shows a summary of modelling results (top), time series of cumulative occurrence of all events, modelled events (green) and events not modelled (red, left). Additionally, this figure provides a vertical sectional view of modelled events (green) with respect to stopes (right). Note that the algorithm excluded some events from the modelled seismic response as they are not spatially clustered and are outside of the sectional view. These events appear to be unrelated to the modelled seismic response due to a significant spatial offset and no indication of time-dependent occurrence. 


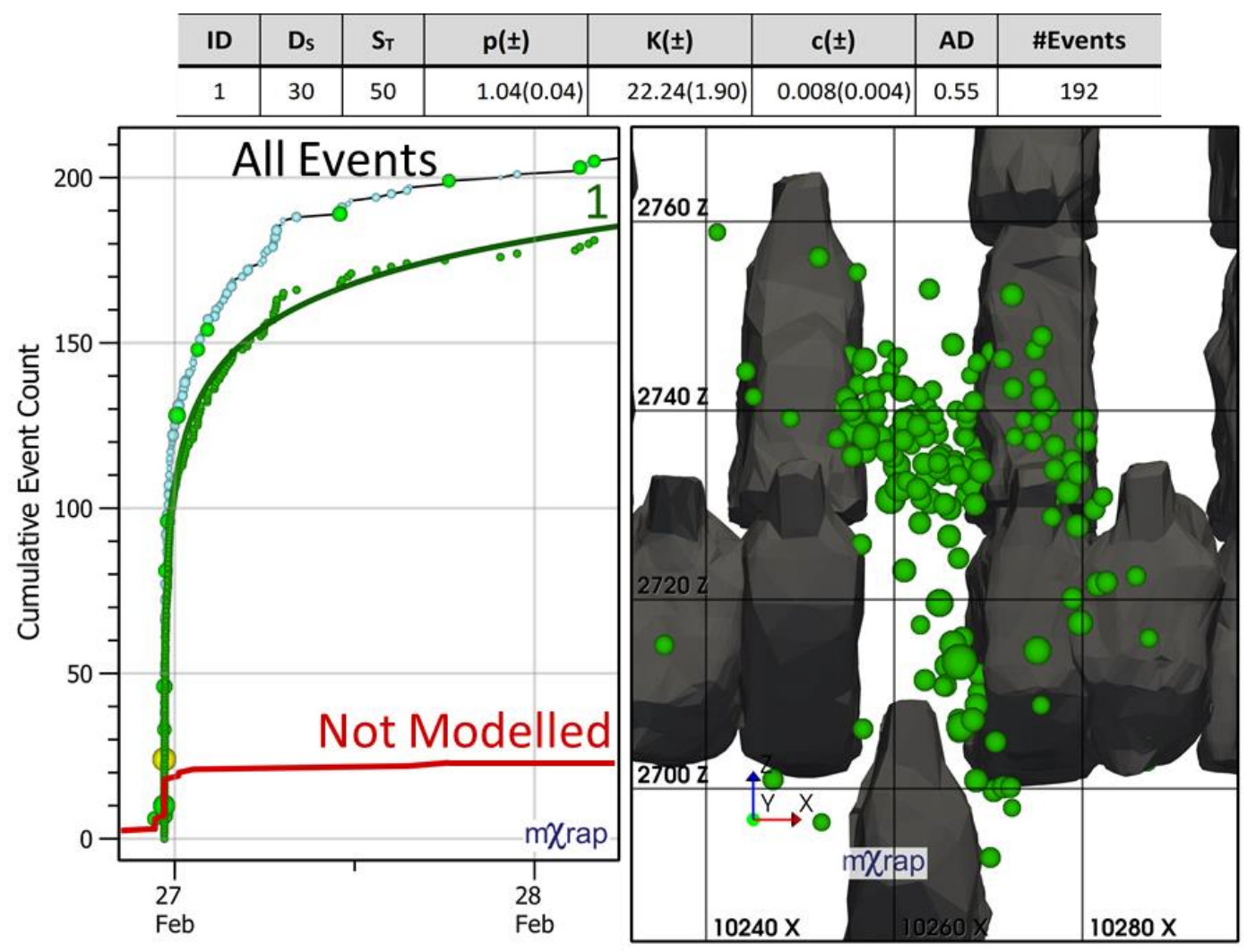

Figure 3 Top: A summary of modelling results. Left: Time series of cumulative occurrence of all events, modelled events (green) and events not modelled (red). Events are sized by local magnitude. Right: a vertical sectional view of modelled events (green) with respect to stope surveys (grey)

The modelling results only consider a dataset of 30 hours after this response, although this seismic response continues well beyond this formative period. In this somewhat exceptional case, due to the time between subsequent blasting, this response can be modelled for an additional 31 days with only minor changes to temporal modelling results and a slight degradation to suitability of fit $(p=1.00, K=23.8, c=0.006$, and $A D=1.12$ ). These parameters are remarkably close to the decay rate and productivity found by modelling of the 30-hour dataset.

The quantification of this response, in terms of the consistency of spatial and temporal occurrence, provides an anecdotal basis for considering these events to be part of a consistent and continuous rock mass failure process. Furthermore, these results imply consistent seismic responses without the influence of additional stress changes (e.g. blasting or large events), the modelling of an early period may provide a forecast of future seismic activity rates. The ability to forecast seismicity activity rates is beneficial when attempting to assess at what time in the future re-entry restrictions could expect to be lifted.

While not the focus of this paper and, hence, not covered in detail, the value of consistent response quantification can be discussed within the conceptual framework of short-term seismic hazard. Accepting the statistical uncertainty of a small dataset and assuming a consistent rock mass failure process, a frequency-magnitude assessment can be considered $(b$-value $=1.54)$. Simplistic seismic hazard analysis suggests that there is a $10 \%$ probability of experiencing an event $M_{L}>2$, given one year exposure to the instantaneous rate of seismicity at one hour after the response began. This probability becomes negligible at the instantaneous rate observed at 10 hours after the response began. The quantification of seismic responses enables an objective approach to the application of re-entry protocols.

A consistent approach to quantification allows for a history of seismic responses that build confidence in nominal parameters given similar mining conditions and ultimately allows for the transparent formulation of protocols with respect to reliability and operational considerations. 


\subsection{Complex seismic responses}

In contrast to the consistent seismic response outlined in Section 3.1, the following example presents analysis results from the delineation of a complex seismic response within an open stope mine over a short time period. Spatial clustering uses a $D_{S}$ of $10 \mathrm{~m}$ and a $S_{T}$ of $50 \%$. This example considers 1,517 events over 3.5 days of seismicity for the mining block and has been delineated into eight seismic responses. Figure 4 shows a summary of modelling results (top), time series of cumulative occurrence of all events, modelled events (sized by magnitude and coloured by response ID), and events not modelled (red, left). Notable large events are annotated by their local magnitude. Additionally, this figure provides a sectional view of modelled events (coloured by response ID) with respect to stopes (right).

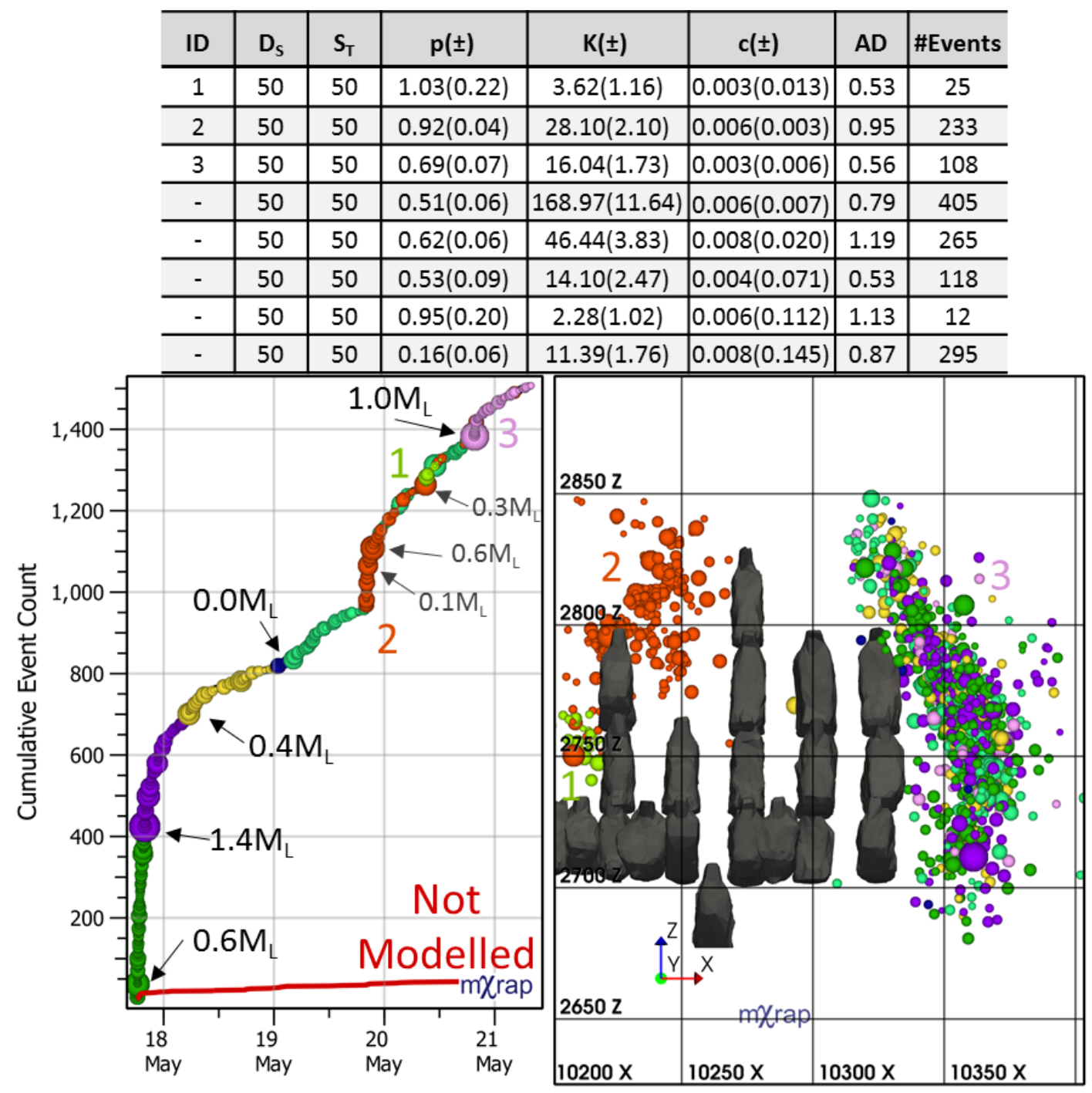

Figure 4 Top: A summary of modelling results. Left: Time series of cumulative occurrence of all events, modelled events (coloured by response ID and sized by local magnitude) and events not modelled (red). Event local magnitudes are annotated. Right: A sectional vertical view of modelled events (coloured by response ID) with respect to stope surveys

Responses 1 and 2 are well modelled by the $\mathrm{MOL}(\mathrm{AD}<2)$ with nominal parameters (i.e. $p \approx 1, \mathrm{~K} \propto \mathrm{N}_{\text {Events, }}$ and $c \approx 0$ ). Response 1 has higher uncertainties due to comprising relatively few events. These responses are located on the left side of the mining block. Of particular interest are the results associated with the larger additional responses on the right side of the mining block. While these responses are adequately modelled, they are quantified by unexpectedly slow decay rates and segment the overall temporal trend in seismicity. The clearest example of this modelling is Response 3 , which occurs on the right abutment of the mining block. 
This response is well modelled by the MOL $(A D=0.56)$ and has a slow decay rate $(p=0.69)$. Relatively slower decay rates are characteristic of the inclusion of previous seismic responses or time-independent seismicity. It is evident from the time series that a large seismic event occurs concurrently with response initiation (late in the time series) and is likely the cause of, or associated with, an additional rock mass failure process that is contributing to seismic productivity. These temporal modelling results appear to be erroneous, although as a consistent approach is implemented to find the optimal temporal solution, these results can potentially indicate a complex rock mass response to mining that warrants further investigation.

It appears that relatively large events $\left(M_{L} \leq 0\right)$ occur with the temporal identification of the seismic response. Furthermore, not all relatively large events $\left(M_{L} \leq 0\right)$ are associated with a new response and this is particularly evident for Response 2, which is spatially unrelated on the left side of the mining block. These observations suggest that increases to seismic productivity are a function of event magnitude and the stability of the rock mass being more sensitive to relatively minor stress changes, which result in a renewed time-dependent stress redistribution process. Such observations, along with additional geotechnical information, may prove valuable when attempting to attribute seismicity to seismic source mechanisms, e.g. time-dependent fault deformation.

Figure 5 examines the cumulative number of events (over time on a log scale) for the erroneous responses that occurred at the beginning of the assessment period (response ID and colours are consistent with Figure 4). This chart illustrates the divergence in temporal characteristics that results in the different responses being delineated early in the time series. Frequency-magnitude analysis was considered to provide additional independent information to support geotechnical interpretations of rock mass failure processes. The b-values for the first response and proceeding two responses were 1.43 and 1.19 respectively. The study of earthquakes suggests that high b-values relate to creeping deformation along a fault, while lower b-values indicate the failure of asperities along the fault (Amelung \& King 1997; Wiemer et al. 1998). A plausible geotechnical hypothesis is that an initial period of time-dependent loading along the fault results in predominately creep deformation (i.e. higher b-value). The failure of asperities along the fault begin to occur once their localised strength is surpassed and the subsequent stress redistribution results in an increased probability of large seismic events (low b-values) and cascading time-dependent behaviour (increased sensitivity of seismic productivity to large events). There is low confidence that this hypothesis reflects reality without considering relevant non-seismic information and observations, and without considering the site-specific conditions that contributed to similar seismic responses in the past.

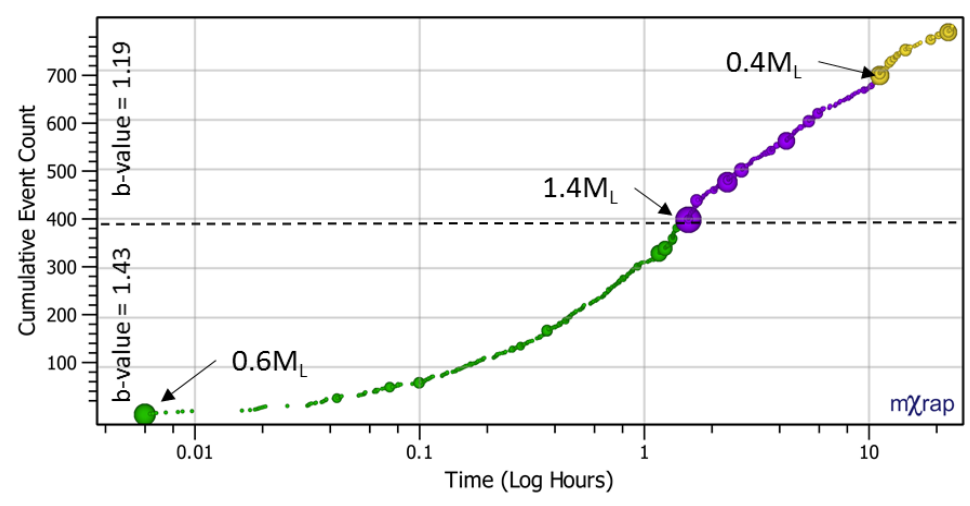

Figure 5 Cumulative events over time (log scale) for the first three responses during this period (coloured by response ID and sized by local magnitude). The $b$-values are annotated for the first response (1.43) and proceeding two responses (1.19)

Irrespective of rock mass failure processes associated with these responses, the identification of unexpected characteristics is important to evaluate the validity of general re-entry protocols and pose research questions such as 'Is seismic response hazard increased if temporal modelling cannot fully represent a seismic response?' 


\subsection{Retrospective mine-wide response assessment}

This retrospective response assessment aims to establish characteristic parameters for different conditions within a sublevel caving mine. The assessment evaluates the components that represent a measure of seismic response hazard. Specifically, K, p and c-parameters, b-values, and modelling intervals.

Seismic responses were identified and delineated in a mine-wide dataset containing 360,000 events. These events were recorded over four years of relatively consistent mining and seismic monitoring. Spatial clustering uses an initial $D_{s}$ of $20 \mathrm{~m}$ for the first iteration of response delineation, then increases this search distance by $20 \mathrm{~m}$ per iteration. All other parameters remain constant per iteration. This assessment uses an $\mathrm{S}_{\text {T }}$ of $10 \%$, a minimum event count to identify a response of 10 events, an identification time window of 15 minutes, and a temporal modelling window of two days. The assessment identified and delineated 1,749 responses containing 96,000 events ( $\approx 25 \%$ of all events). Only responses with reasonable parameters were considered in subsequent analyses by removing responses that were unsuitably modelled by the MOL (AD $>2)$, unreasonably short $\left(T_{E}<0.5 \mathrm{~h}\right.$ ), exhibited high uncertainty in parameters ( $\left.\mathrm{SE}_{\mathrm{AVE}}>100 \%\right)$, or had high c-parameters $(\mathrm{c}>0.1 \mathrm{~h})$. This filtering removed 8,500 events associated with 190 responses from further consideration.

Seismic responses are spatially allocated to unique geotechnical domains. Geotechnical domains are qualitatively defined with respect to the nature of mining (e.g. direction of mining, and development versus established production) along with generalised geological and geotechnical information. Each was given an arbitrary ID in Table 1, which corresponds to the associated figures. This table provides quantification of mean parameters from temporal modelling. Each spatial geotechnical domain has distinctly coloured markers in Figure 6 and is annotated by the corresponding numerical ID (top: plan view; bottom: Y-section). Each sphere in this figure represents a single seismic response, each response comprises of $\approx 60$ seismic events, on average. The same marker colours correspond to the cumulative density functions of K-parameters (left) and p-parameters (right) for each geotechnical domain (Figure 7).

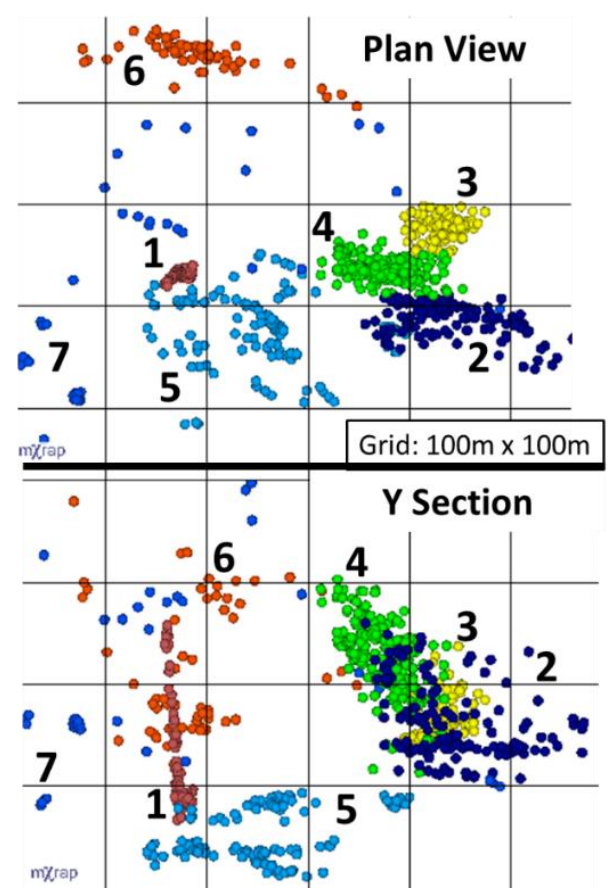

Figure 6 Spatial locations of geotechnical domains are shown on a plan view (top) and Y-section (bottom). Each sphere is an individual seismic response 

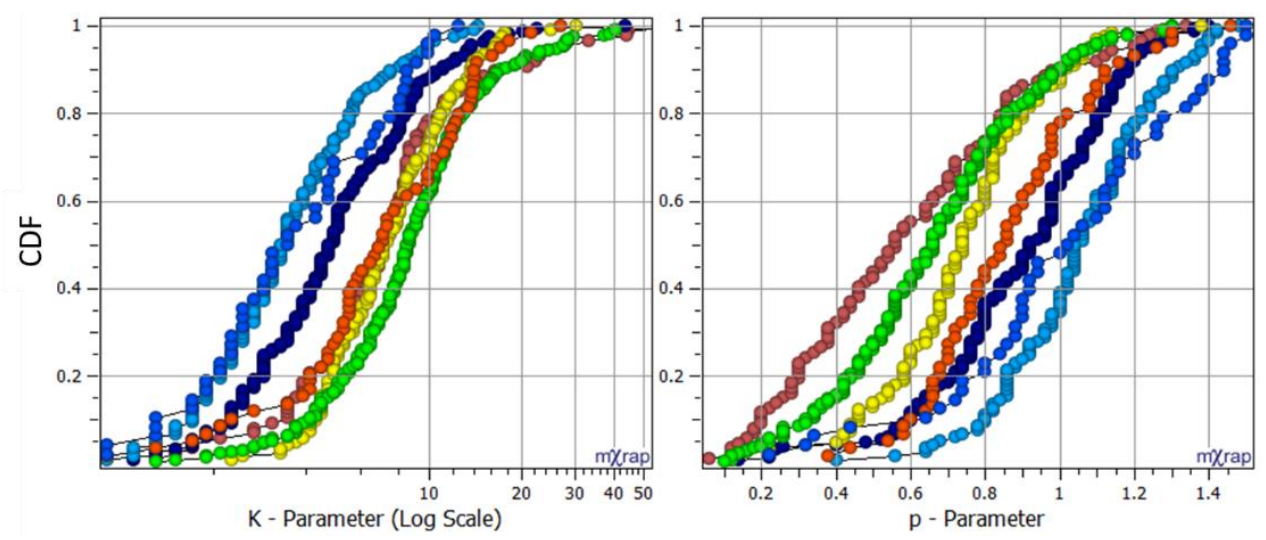

Figure 7 Cumulative density functions per geotechnical domain for the K-parameter (left) and p-parameter (right). Markers are coloured corresponding to the spatial plot on Figure 6

Table 1 The geotechnical domain, number of responses in each domain (count), characteristics, and relevant mean parameters for temporal quantification. The b-value of each domain is also assessed

\begin{tabular}{|c|c|c|c|c|c|c|c|c|}
\hline Domain description & ID & $\begin{array}{l}\text { Response } \\
\text { count }\end{array}$ & $\mathbf{T}_{\mathrm{S}}$ & $\mathbf{T}_{\mathbf{E}}$ & $\mathbf{p}( \pm)$ & $\mathbf{K}( \pm)$ & $\mathbf{c}( \pm)$ & $\mathbf{b}( \pm)$ \\
\hline $\begin{array}{l}\text { Raise bore } \\
\text { development under } \\
\text { high stress conditions }\end{array}$ & 1 & 136 & 0.032 & 12.7 & $\begin{array}{c}0.65 \\
(0.38)\end{array}$ & $\begin{array}{c}11.1 \\
(11.8)\end{array}$ & $\begin{array}{c}0.00 \\
(0.01)\end{array}$ & $\begin{array}{c}1.40 \\
(0.02)\end{array}$ \\
\hline $\begin{array}{l}\text { Development blasting, } \\
\text { faulting, and } \\
\text { contrasting rock } \\
\text { properties }\end{array}$ & 2 & 315 & 0.019 & 11.6 & $\begin{array}{c}0.86 \\
(0.29)\end{array}$ & $\begin{array}{c}7.3 \\
(5.8)\end{array}$ & $\begin{array}{c}0.01 \\
(0.02)\end{array}$ & $\begin{array}{c}0.99 \\
(0.02)\end{array}$ \\
\hline $\begin{array}{l}\text { Development and } \\
\text { production blasting, } \\
\text { and geologically } \\
\text { controlled stress } \\
\text { conditions }\end{array}$ & 3 & 205 & 0.020 & 19.0 & $\begin{array}{c}0.74 \\
(0.27)\end{array}$ & $\begin{array}{c}8.4 \\
(4.2)\end{array}$ & $\begin{array}{c}0.01 \\
(0.02)\end{array}$ & $\begin{array}{c}1.00 \\
(0.01)\end{array}$ \\
\hline $\begin{array}{l}\text { Development and } \\
\text { production blasting, } \\
\text { geological features, } \\
\text { and crosscuts principal } \\
\text { stress }\end{array}$ & 4 & 420 & 0.023 & 20.0 & $\begin{array}{c}0.64 \\
(0.29)\end{array}$ & $\begin{array}{c}11.6 \\
(10.0)\end{array}$ & $\begin{array}{c}0.01 \\
(0.02)\end{array}$ & $\begin{array}{c}1.21 \\
(0.01)\end{array}$ \\
\hline $\begin{array}{l}\text { Development blasting } \\
\text { under high stress } \\
\text { conditions due to } \\
\text { increased depth }\end{array}$ & 5 & 297 & 0.015 & 9.2 & $\begin{array}{c}0.99 \\
(0.28)\end{array}$ & $\begin{array}{l}5.6 \\
(4.1)\end{array}$ & $\begin{array}{c}0.01 \\
(0.02)\end{array}$ & $\begin{array}{c}1.42 \\
(0.03)\end{array}$ \\
\hline $\begin{array}{l}\text { Development and } \\
\text { production blasting, } \\
\text { geological features, } \\
\text { and varied stress } \\
\text { conditions }\end{array}$ & 6 & 91 & 0.013 & 13.7 & $\begin{array}{c}0.86 \\
(0.26)\end{array}$ & $\begin{array}{l}9.0 \\
(7.0)\end{array}$ & $\begin{array}{c}0.01 \\
(0.03)\end{array}$ & $\begin{array}{c}0.99 \\
(0.02)\end{array}$ \\
\hline $\begin{array}{l}\text { Isolated responses to } \\
\text { development blasting }\end{array}$ & 7 & 95 & 0.027 & 9.7 & $\begin{array}{c}0.95 \\
(0.32)\end{array}$ & $\begin{array}{c}5.4 \\
(3.8)\end{array}$ & $\begin{array}{c}0.00 \\
(0.01)\end{array}$ & $\begin{array}{l}1.35 \\
(0.05)\end{array}$ \\
\hline
\end{tabular}


Consideration of the distribution of response quantification with respect to specific geotechnical domains allows the following qualitative observations to be made:

- Seismicity associated with raise boring has distinctive low p-parameters and a distribution of K-parameters comparable to the most productive responses $(3,4,6)$.

- Domains that only contain development blasting result in higher p-parameters and lower K-parameters (light blue (5), blue (7), and dark blue (2)).

- Geotechnical domains with high stress conditions, i.e. deeper in the mine (light blue (5)), or crosscutting principal stress directions (green (4)), have a relatively higher b-value.

- Domains with lower b-values are likely influenced by geological features (Legge \& Spottiswoode 1987) and also have lower p-parameters, and higher K-parameters (dark blue (2) versus light blue (5)).

- Domains that likely contain a mixture of influences (stress concentration, geology, production, and development blasting) have lower p-parameters and higher K-parameters (green (4), yellow (3), orange (6)).

Quantifying seismic responses through the mining environment is essential to optimising the management of seismic response hazard. The identification and delineation of seismic responses provides historical characterisations of geotechnical domains and provides guidance for future responses in these regions. Retrospective quantification also provides a reference to assess when seismic responses are uncharacteristic of a geotechnical domain. For example, when geology begins to influence seismic responses to deeper development (and production), it can be expected that the productivity of responses will increase, the temporal decay of event occurrence will decrease, and there will be more large events relative to small events.

\section{Conclusion}

The identification and delineation of seismic responses allows the consistent quantification of spatially-clustered, time-dependent seismicity. This method enables the following outcomes:

- The ability to statistically assess if an individual seismic response is consistent in space and time provides a basis for assessing if events are due to a continuous rock mass failure process. These observations may provide quantitate information when attempting to assign seismicity to specific seismic source mechanisms.

- The quantification of nominal response parameters, which is an important aspect for the formulation of transparent re-entry protocols and assessing when re-entry protocols are not applicable to uncharacteristic responses.

- The quantification of seismic responses enables an objective approach to the application of re-entry protocols within the probabilistic framework of short-term seismic hazard.

- Assessment of historical seismic responses builds confidence in nominal parameters for geotechnical domains and provides guidance for future responses in these regions under similar mining conditions and a reference for assessing if a seismic response is uncharacteristic.

\section{Acknowledgement}

This research is part of Phase 5 of the Australian Centre for Geomechanics' Mine Seismicity and Rockburst Risk Management project, sponsored by the following organisations: Barrick Gold of Australia, BHP Billiton Nickel West, BHP Billiton Olympic Dam, Independence Group (Lightning Nickel), LKAB Sweden, Perilya Limited (Broken Hill Mine), Vale Inc. Canada, Agnico-Eagle Canada, Gold Fields St Ives Gold Operations, Hecla USA, Kirkland Lake Gold, MMG Golden Grove, Newcrest Cadia Valley Operations, Newmont Asia Pacific, Xstrata Copper (Kidd Mine), Xstrata Nickel Rim, and the Minerals and Energy Research Institute of Western Australia. 


\section{References}

Akaike, H 1974, 'A new look at the statistical model identification', IEEE Transactions on Automatic Control, vol. 19, no. 6, pp. 716-723.

Amelung, F \& King, G 1997, 'Earthquake scaling laws for creeping and non-creeping faults', Geophysical Research Letters, vol. 24, no. 5, pp. 507-510.

Anderson, TW \& Darling, DA 1954, 'A test of goodness of fit', American Statistical Association, vol. 49, no. 268, pp. 765-769.

Cho, NF, Tiampo, KF, McKinnon, SD, Vallejos, JA, Klein, W \& Dominguez, R 2010, 'A simple metric to quantify seismicity clustering', Nonlinear Processes in Geophysics, vol. 17, no. 4, pp. 293-302.

Dieterich, J 1994, 'A constitutive law for rate of earthquake production and its application to earthquake clustering', Geophysical Research: Solid Earth (1978-2012), vol. 99, no. B2, pp. 2601-2618.

Enescu, B, Mori, J, Miyazawa, M \& Kano, Y 2009, 'Omori-Utsu Law c-values associated with recent moderate earthquakes in Japan', Bulletin of the Seismological Society of America, vol. 99, no. 2A, pp. 884-891.

Eremenko, VA, Eremenko, AA, Rasheva, SV \& Turuntaev, SB 2009, 'Blasting and the man-made seismicity in the Tashtagol mining area', Mining Science, vol. 45, no. 5, pp. 468-474.

Ester, M, Kriegel, H, Sander, J \& Xu, X 1996, 'A density-based algorithm for discovering clusters in large spatial databases with noise', Knowledge Discovery and Data Mining, vol. 96, no. 34, pp. 226-231.

Falmagne, V 2001, Quantification of rock mass degradation using micro-seismic monitoring and applications for mine design, PhD thesis, Queen's University, Kingston.

Frohlich, C \& Davis, SD 1990, 'Single-link cluster analysis as a method to evaluate spatial and temporal properties of earthquake catalogues', Geophysical Journal International, vol. 100, no. 1, pp. 19-32.

Gasperini, P \& Lolli, B 2006, 'Correlation between the parameters of the aftershock rate equation: Implications for the forecasting of future sequences', Physics of the Earth and Planetary Interiors, vol. 156, no. 1, pp. 41-58.

Gasperini, P \& Lolli, B 2009, 'An empirical comparison among aftershock decay models', Physics of the Earth and Planetary Interiors, vol. 175 , no. 3 , pp. 183-193.

Gross, SJ \& Kisslinger, C 1994, 'Tests of models of aftershock rate decay', Bulletin of the Seismological Society of America, vol. 84, no 5, pp. 1571-1579.

Heal, D 2007, 'Perilya Broken Hill - investigation of re-entry times following production blasts', Mine Seismicity and Rockburst Risk Management Project, Australian Centre for Geomechanics, Perth.

Helmstetter, A, Kagan, YY \& Jackson, DD 2005, 'Importance of small earthquakes for stress transfers and earthquake triggering', Geophysical Research: Solid Earth (1978-2012), vol. 110, no. B5, pp. 1-13.

Hills, PB \& Penney, AR 2008, 'Management of seismicity at the Beaconsfield gold mine, Tasmania', Proceedings of the 10th Underground Operators' Conference, Australasian Institute of Mining and Metallurgy, Carlton South, pp. 157-170.

Hudyma, M 2008, Analysis and Interpretation of Clusters of Seismic Events in Mines, PhD thesis, The University of Western Australia, Perth.

Jain, AK, Murty, MN \& Flynn, PJ 1999, 'Data clustering: a review', ACM Computing Surveys, vol. 31, no. 3, pp. 264-323.

Kagan, YY 2004, 'Short-term properties of earthquake catalogs and models of earthquake source', Bulletin of the Seismological Society of America, vol. 94, no. 4, pp. 1207-1228.

Kagan, YY 2006, 'Why does theoretical physics fail to explain and predict earthquake occurrence?', in P Bhattacharyya \& BK Chakrabarti (eds), Modelling Critical and Catastrophic Phenomena in Geoscience: A Statistical Physics Approach, Springer-Verlag Berlin Heidelberg, Heidelberg, pp. 303-359.

Kagan, YY \& Jackson, DD 1991, 'Long-term earthquake clustering', Geophysical Journal International, vol. 104, no. 1, pp. 117-133.

Kgarume, T 2010, Mine Aftershocks and Implications for Seismic Hazard Assessment, MSc thesis, University of the Witwatersrand, Johannesburg.

Kgarume, T, Spottiswoode, S \& Durrheim, R 2010a, 'Statistical properties of mine tremor aftershocks', Pure and Applied Geophysics, vol. 167 , no. 1 , pp. 107-117.

Kgarume, TE, Spottiswoode, SM \& Durrheim, RJ 2010b, 'Deterministic properties of mine tremor aftershocks', in M Van Sint Jan \& Y Potvin (eds), 5th International Seminar on Deep and High Stress Mining, Australian Centre for Geomechanics, Perth, pp. 227-237.

Kisslinger, C 1993, 'The stretched exponential function as an alternative model for aftershock decay rate', Geophysical Research: Solid Earth (1978-2012), vol. 98, no. B2, pp. 1913-1921.

Kriegel, H, Kröger, P, Sander, J \& Zimek, A 2011, 'Density-based clustering', Wiley Interdisciplinary Reviews: Data Mining and Knowledge Discovery, vol. 1, no. 3, pp. 231-240.

Kwiatek, G 2004, 'A search for sequences of mining-induced seismic events at the Rudna Copper Mine in Poland', Acta Geophysica Polonica, vol. 52, no. 2, pp. 115-171.

Larsson, K 2004, 'Mining induced seismicity in Sweden', Licentiate, Luleå University of Technology, Luleå.

Legge, NB \& Spottiswoode, SM 1987, 'Fracturing and microseismicity ahead of a deep gold mine stope in the pre-remnant and remnant stages of mining', in G Herget \& S Vongpaisal (eds), Proceedings of the 6th International Congress on Rock Mechanics, Balkema, Rotterdam, pp. 1071-1077.

Lewis, PAW 1961, 'Distribution of the Anderson-Darling statistic', The Annals of Mathematical Statistics, vol. 32, no. 4, pp. 1118-1124. 
Malek, F \& Leslie, IS 2006, 'Using seismic data for rockburst re-entry protocol at INCO's Copper Cliff North Mine', in D Yale, S Holtz, C Breeds \& U Ozbay (eds), Proceedings of the 41st U.S. Symposium on Rock Mechanics, American Rock Mechanics Association, Alexandria, pp. 1-10.

Melick, AG 2007, Beaconsfield Investigation Report, Tasmanian Government, viewed 9 January 2017, http://www.magistratescourt.tas.gov.au/_data/assets/pdf_file/0005/120884/alx.016.002.0002_001.pdf

Mendecki, AJ 2005, 'Persistence of seismic rock mass response to mining', in Y Potvin \& M Hudyma (eds), Proceedings of the 6th International Symposium on Rockburst and Seismicity in Mines, Australian Centre for Geomechanics, Perth, pp. 97-105.

Mendecki, AJ 2008, 'Keynote address: forecasting seismic hazard in mines', in Y Potvin, J Carter, A Dyskin \& R Jeffrey (eds), 1st Southern Hemisphere International Rock Mechanics Symposium, Australian Centre for Geomechanics, Perth, pp. 1-17.

Mendecki, AJ \& Lynch, RA 2004, GAP601a: Experimental and theoretical investigations of fundamental processes in mining induced fracturing and rock instability close to excavations, ISS International Limited, Johannesburg.

Molchan, GM \& Dmitrieva, OE 1992, 'Aftershock identification - methods and new approaches', Geophysical Journal International, vol. 109, no. 3, pp. 501-516.

Narteau, C, Shebalin, P \& Holschneider, M 2002, 'Temporal limits of the power law aftershock decay rate', Geophysical Research: Solid Earth (1978-2012), vol. 107, no. B12, pp. 1-12.

Nyffenegger, P 1998, Aftershock occurrence rate decay for individual sequences and catalogs, PhD thesis, University of Texas at Austin, Austin.

Nyffenegger, P \& Frohlich, C 1998, 'Recommendations for determining $p$ values for aftershock sequences and catalogs', Bulletin of the Seismological Society of America, vol. 88, no. 5, pp. 1144-1154.

Nyffenegger, P \& Frohlich, C 2000, 'Aftershock occurrence rate decay properties for intermediate and deep earthquake sequences', Geophysical Research Letters, vol. 27, no. 8, pp. 1215-1218.

Ogata, Y 1983, 'Estimation of the parameters in the modified Omori formula for aftershock frequencies by the maximum likelihood procedure', Physics of the Earth, vol. 31, no. 2. pp. 115-124.

Omori, F 1894a, 'On after-shocks', Seismological Journal of Japan, vol. 19, pp. 71-80.

Omori, F 1894b, 'On the after-shocks of earthquakes', Journal of the College of Science, Imperial University of Tokyo, vol. 7, no. 2, pp. 111-200.

Penney, AR 2011, Development Of Re-Entry Guidelines And Exclusion Zones At The Tasmania Gold Mine, MSc thesis, Curtin University of Technology, Perth.

Plenkers, K, Kwiatek, G, Nakatani, M \& Dresen, G 2010, 'Observation of seismic events with frequencies $\mathrm{f}>25 \mathrm{kHz}$ at Mponeng deep gold mine, South Africa', Seismological Research Letters, vol. 81, no. 3, pp. 467-479.

Rebuli, DB \& Kohler, SJ 2014, 'Using clustering algorithms to assist short-term seismic hazard analysis in deep South African mines', in M Hudyma \& Y Potvin (eds), Proceedings of the Seventh International Seminar on Deep and High Stress Mining, Australian Centre for Geomechanics, Perth, pp. 107-121.

Spottiswoode, SM 2000, 'Aftershocks and foreshocks of mine seismic events', Proceedings of the 3rd International Workshop on the Application of Geophysics to Rock and Soil Engineering, International Society for Rock Mechanics, Lisboa, pp. 82-88.

Utsu, T 1961, 'A statistical study of the occurrence of aftershocks', Geophysical Magazine, vol. 30, no. 4. pp. 521-605.

Utsu, T 2002, 'Statistical features of seismicity', International Geophysics Series, vol. 81, no. A, pp. 719-732.

Utsu, T, Ogata, Y \& Matsu'ura, RS 1995, 'The centenary of the Omori formula for a decay law of aftershock activity', Physics of the Earth, vol. 43, no. 1, pp. 1-33.

Vallejos, JA \& McKinnon, SD 2008, 'Guidelines for development of re-entry protocols in seismically active mines', Proceedings of the 42 nd U.S. Symposium on Rock Mechanics, American Rock Mechanics Association, Alexandria, Paper 08-097.

Vallejos, JA \& McKinnon, SD 2010a, 'Omori's law applied to mining-induced seismicity and re-entry protocol development', Pure and Applied Geophysics, vol. 167, no. 1-2, pp. 91-106.

Vallejos, JA \& Mckinnon, SD 2010b, 'Temporal evolution of aftershock sequences for re-entry protocol development in seismically active mines', in M Van Sint Jan \& Y Potvin (eds), Proceedings of the 5th International Seminar on Deep and High Stress Mining, Australian Centre for Geomechanics, Perth, pp. 199-214.

Vallejos, JA \& McKinnon, SD 2011, 'Correlations between mining and seismicity for re-entry protocol development', International Journal of Rock Mechanics and Mining Sciences, vol. 48, no. 4, pp. 616-625.

Wesseloo, J 2014, 'Evaluation of the spatial variation of the b-value', Journal of the South African Institute of Mining and Metallurgy, vol. 114 , no. 5, pp. 823-828.

Wiemer, S, McNutt, SR \& Wyss, M 1998, 'Temporal and three-dimensional spatial analyses of the frequency-magnitude distribution near Long Valley Caldera, California', Geophysical Journal International, vol. 134, no. 2, pp. 409-421.

Woodward, K 2015, Identification and delineation of mining induced seismic responses, PhD thesis, The University of Western Australia, Perth. 
